\title{
Ganzheitsmedizin aktuell
}

Schweiz Z Ganzheitsmed 23 | 5 | 11

Neu: Sinupret ${ }^{\circledR}$ Sirup - ab 2 Jahren

Die neue kassenpflichtige Darreichungsform des bewährten pflanzlichen Arzneimittels Sinupret ${ }^{\circledR}$ ist speziell für Kinder geeignet: ab 2 Jahren, guter Geschmack, ohne Farbstoffe, zahnschonend. Mit der Einführung von Sinupret $^{\circledR}$ Sirup ergänzt die Biomed AG das Sortiment

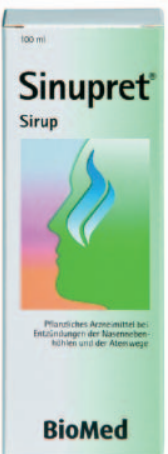
ihres erfolgreichen phytotherapeutischen Arzneimittels Sinupret um eine weitere Darreichungsform. Sinupret ${ }^{\circledR}$ Sirup ist insbesondere für Kinder ab 2 Jahren geeignet: Er hat einen angenehmen Geschmack und erhöht damit die Compliance der kleinen Patienten, die Arzneimittel nicht selten wegen eines unange- nehmen Geschmacks ablehnen. Sinupret ${ }^{\circledR}$ Sirup ist ausserdem frei von Farbstoffen, enthält keinen Zucker und ist somit zahnschonend.

Die Extrakte aus Enzianwurzel, Schlüsselblumenblüten, Eisenkraut, Holunderblüten und Sauerampferkraut wirken sekretolytisch, antiphlogistisch, antiviral und antibakteriell [1].

Mit diesem Wirkspektrum ist Sinupret ${ }^{\circledR}$ effektiv bei akuten und chronischen Entzündungen der Nasennebenhöhlen und der Atemwege. Zugleich beugt es einer Verschlimmerung oder Chronifizierung der Sinusitis vor.

Sinupret ${ }^{\circledR}$ Sirup ist in der Packungsgrösse $100 \mathrm{ml}$ erhältlich, gehört der Liste C an und ist kassenpflichtig.

\section{Referenzen}

März RW et al.: Wirkprofil und Wirksamkeit eines pflanzlichen Kombinationspräparates zur Behandlung der Sinusitis. Wien Med Wochenschr 1999;149:202-208.
Gekürzte Fachinformation Sinupret ${ }^{\circledR}$ Sirup (pflanzliches Arzneimittel)

Z: Enzianwurzel, Schlüsselblumenblüten, Sauerampferkraut, Holunderblüten, Eisenkraut. I: Entzündungen von Nasennebenhöhlen und Atemwegen. D: $\geq 12 \mathrm{~J} .: 3 \times \mathrm{tgl}$. $7,0 \mathrm{ml} ; \geq 6$ J.: $3 \times$ tgl. $3,5 \mathrm{ml} ; \geq 2 \mathrm{~J} .: 3 \times$ tgl. $2,1 \mathrm{ml}$. Schwangerschaft: Über die Anwendung entscheidet der Arzt. KI: Überempfindlichkeit auf einen der Inhaltsstoffe. UW: Überempfindlichkeitsreaktionen. P: Sinupret ${ }^{\circledR}$ Sirup $100 \mathrm{ml}^{*}$. Warnhinweis: Enthält 8 Vol.-\% Alkohol. Liste C. Ausführliche Angaben siehe Arzneimittel-Kompendium der Schweiz. *kassenpflichtig

Weitere Informationen bei

Biomed AG

www.biomed.ch

Tel. +41 4480216-27, Fax -00

biomed@biomed.ch

\section{Produktneuheit!}

Magnesium-Diasporal activ - jetzt als Brausetablette!

Ab sofort gibt es Diasporal activ auch als frei verkäufliches Nahrungsergänzungsmittel: Magnesium-Diasporal activ Brausetablette. Das ideale Produkt für alle aktiven Menschen im Beruf und in der Freizeit, die einen erhöhten Magnesium- und Flüssigkeitsbedarf

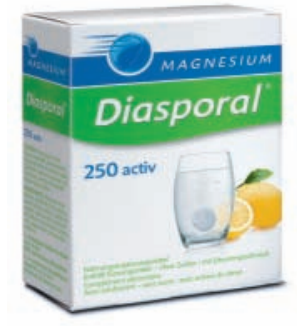
haben. Das Besondere: Es enthält Magnesiumcitrat, wie es als Baustein im Körper vorkommt, und unterstützt so wichtige Muskel- und Nervenfunktionen.
Magnesium-Diasporal activ 250:

Darreichungsform: Brausetabletten

Einnahme: schnell und klar zum Auflösen in Flüssigkeit

Einnahmehäufigkeit: $1 \times$ täglich und schnell aktiv

Wirkstoff: körperfreundliches Magnesiumcitrat

Dosierung: $250 \mathrm{mg}$

Geschmack: frischer Zitronengeschmack

Inhaltsstoffe: natürlich ohne Zucker, ohne künstliche Farbstoffe, ohne Gluten/ Lactose

Packungsgrösse: 20er Packung

(Pharmacode: 4862299), 60er Packung (Pharmacode: 4862282).

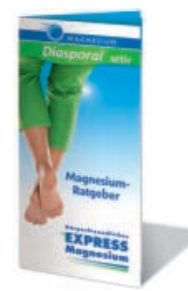

Neu: Magnesium-Ratgeber - jetzt gratis anfordern $\mathrm{Ab}$ sofort gibt es für Patienten einen neuen Magnesium -Ratgeber mit viel kompaktem Wissen sowie leicht verständlichen Antworten auf die häufigsten Fragen, z.B. «Stimmt Ihr Magnesiumhaushalt? Wie muss eine sinnvolle Magnesiumdosierung aussehen oder was muss ich bei der Einnahme beachten?»

Weitere Informationen bei

Doetsch Grether AG

Infoline: +41 800804042 (gebührenfrei)

www.diasporal.ch

Bestellungen:

Tel. +41 34 42146-46, Fax -12

bestellungen@alloga.ch

\section{Ticker+++ Ticker+++ Ticker+++ Ticker+++ Ticker+++ Ticker+++ Ticker+++}

Schaper \& Brümmer GmbH \& Co. KG. Bei schwachen Venen hat sich die Heilkraft der Rosskastanie (z.B. in Venalot ${ }^{\circledR}$, Apotheke) bewährt. Venalot ${ }^{\circledR}$ mindert das Druckgefühl in den Beinen. Die Schwellungen lassen spürbar nach, Schmerzen und nächtliche Wadenkrämpfe werden gemindert. Dank des natürlichen Rosskastaniensamenextraktes werden die Blutgefässe effektiv gestärkt und die Beschwerden der Venenschwäche reduziert.

Schaper \& Brümmer GmbH \& Co. KG

Tel. +49 5341 307-0

www.schaper-bruemmer.de
Natürlich Gesund e.V. Der Granatapfel wird immer beliebter. Eine Flut von Produkten, ob als Saft, Konzentrat oder Kapsel, steht dem Verbraucher zur Verfügung. Um die Entscheidung zu erleichtern, hat der Verband "Natürlich Gesund» e.V. eine Produktstudie bei der Universität Hohenheim in Auftrag gegeben. Ihr Ergebnis: Es kommt vor allem auf die Zusammensetzung der Polyphenole an. Der Produkttest und ein umfassender Fachratgeber können bei dem Verband gegen Rechnung angefordert werden.

Natürlich Gesund e.V.

Tel. +49 180588077730

www.granatapfelsaft.de
Chinesische Naturheilkunde Akademie e.V. Die Chinesische Regierung gibt einmalig ein Zuschussstipendium zum «Doctor of Medicine»Studium für berufstätige Ärzte, Physiotherapeuten etc. mit Master-Degree. Die TCM-Universität Shandong bietet folgende Studiengänge in deutscher Sprache mit Fachdolmetschern an: TCM Surgery, TCM Gynecology, CM OsteoTraumatology, TCM Pediatrics sowie Acupuncture \& Tuina. Beginn 2012, Anmeldung jetzt schon möglich.

Chinesische Naturheilkunde Akademie e.V.

Tel. +49 922184100

www.chinesische-naturheilkunde.de

\section{KARGER}

